\title{
Morfometria testicular durante o ciclo reprodutivo de Dendropsophus minutus (Peters) (Anura, Hylidae)
}

\author{
Lia R. de S. Santos \& Classius de Oliveira
}

${ }^{1}$ Programa de Pós-graduação em Biologia Animal, Departamento de Biologia, Instituto de Biociências, Letras e Ciências
Exatas, Universidade Estadual Paulista. 15040-000 São José do Rio Preto, São Paulo, Brasil. E-mail: classius@ibilce.unesp.br

ABSTRACT. Testicular morphometry during the reproductive cycle of Dendropsophus minutus (Peters) (Anura, Hylidae). This study describes the male reproductive cycle of Dendropsophus minutus (Peters, 1872) based on testicular morphometric analysis, related to climatic conditions. Fifty individuals where collected in São José do Rio Preto, São Paulo. After macroscopic analysis, the testes were submitted to histological routine, fixed with Bouin, embedded in historesin. Sections of $2 \mu \mathrm{m}$ were stained with $1 \%$ toluidine blue and observed to the microscope. The testes of $D$. minutus are small organs (length $1.90 \pm 0.13 \mathrm{~mm}$ ), whitish, oviform and found in the abdominal cavity. They are located in the cranial extremity of the kidneys and present asymmetry as far as position. According to statistical analyses, there is no intra-individual variation in length and weight of the testes, as well as in the area and diameter of the seminiferous locules. Concerning the testicular histology, it was possible to identify, throughout the year, in seminiferous locules, all the cellular types from the spermatogenic lineage, characterizing a continuous gametogenesis, corroborated by ecological and behavioral factors. Information about testicular morphometry and reproductive cycle has important biological value for anurans in the neotropics.

KEY WORDS. Anuran; reproduction; spermatogenesis; testis.

RESUMO. Este estudo descreve o ciclo reprodutivo de machos de Dendropsophus minutus (Peters, 1872) com base na análise da morfometria testicular e a correlação com parâmetros climáticos. Cinqüenta indivíduos foram coletados em São José do Rio Preto, São Paulo. Após as análises macroscópicas, os testículos foram encaminhados à rotina histológica, fixados com Bouin e incluídos em historesina. Cortes de $2 \mu \mathrm{m}$ foram corados com azul de toluidina $1 \%$ e observados ao microscópio. Testículos de $D$. minutus são órgãos pequenos (comprimento $1,90 \pm 0,13 \mathrm{~mm}$ ), esbranquiçados, com forma oval e encontrados na cavidade abdominal. Estão localizados na extremidade cranial dos rins e apresentam assimetria quanto a sua posição. Estatisticamente não há variação intra-individual no comprimento e no peso dos testículos, bem como na área e diâmetro dos lóculos seminíferos. Quanto à histologia testicular, foi possível identificar ao longo do ano nos lóculos seminíferos, todos os tipos celulares da linhagem espermatogênica, caracterizando uma gametogênese contínua, corroborada por fatores ecológicos e comportamentais. Informações sobre a morfometria testicular e ciclo reprodutivo tem importante valor biológico para anuros de regiões neotropicais.

PALAVRAS-CHAVE. Anuro; espermatogênese; reprodução; testículo.

O sucesso da dispersão dos vertebrados depende, entre outras, da adaptação de seus órgãos e ciclos reprodutivos ao ambiente no qual eles estão envolvidos. O controle dos ciclos reprodutivos requer que os organismos sejam capazes de detectar as mudanças no ambiente e ajustar os parâmetros fisiológicos de acordo com as condições necessárias, isto é realizado através de modulação do sistema neuroendócrino (CALLARD et al. 1978). De modo geral, a atividade reprodutiva de anuros de regiões tropicais sazonais é associada ao período úmido (Hoogmoed \& Gorzula 1979). Nestes locais, os processos ecológicos anuais tais como pluviosidade e temperatura são vitais para assegurar a reprodução da maioria das espécies (HOOGMOED \& Gorzula 1979, Aichinger 1987). Estas variáveis determinam a época favorável do ano e a duração em que os anuros se mantém ativos, determinando sua distribuição estacional (DuelLMAN \& Trueb 1994, HuANG et al. 1997).

Tem sido sugerido que o controle da espermatogênese é mantido pela interação entre ritmos endógenos e fatores externos (Paniagua et al. 1990, SAsso-Cerri et al. 2004). A temperatura e o fotoperíodo são os principais fatores ambientais controladores de ciclos reprodutivos sazonais em anfíbios (Lorts 1974, Rastogi et al. 1976, Paniagua et al. 1990). Mudanças ambientais 
nesses fatores podem interferir na espermatogênese e estabelecer ciclos gametogênicos do tipo contínuo, descontínuo ou potencialmente contínuo (Lofts 1974, HuANG et al. 1997).

Os tipos descontínuos são comumente encontrados em espécies de zonas temperadas e geralmente têm um discreto período de reprodução com pronunciadas mudanças no tamanho das gônadas, na produção de gametas e nas estruturas sexuais acessórias. No tipo potencialmente contínuo ocorre uma interrupção parcial na atividade espermatogênica durante algumas estações no ano, mas espermatogônias primárias permanecem sensíveis à estimulação gonadotrófica. Espécies que habitam regiões tropicais, normalmente apresentam o tipo contínuo de ciclo reprodutivo.

De modo geral, os estudos sobre aspectos reprodutivos dos anuros hilídeos descrevem principalmente o comportamento reprodutivo (HADDAD \& SAWAYA 2000, Bastos \& HADDAD 2002), sendo poucos os estudos anatômicos descrevendo o sistema reprodutor (Oliveira \& Vicentini 1998, Oliveira \& SAntos 2004, OliveIRA \& ZierI 2005) com ênfase na morfologia gonadal. Entretanto, os trabalhos com Scinax ranki (Andrade \& Cardoso, 1987) de TABOGA \& Dolder (1991) e com Scinax fuscovarius (Lutz, 1925) de Oliveira et al. (2003) descrevem características histológicas dos testículos. Estudos contendo informações sobre os ciclos reprodutivos com ênfase em análise morfológica foram realizados em espécies de outras famílias, como em Lithobates catesbeianus (Shaw, 1802) (SAsso-CerRI et al. 2004).

Estetrabal ho descreve o ciclo reprodutivo de Dendropsophus minutus com base em análises morfológicas e morfométricas. Utilizando-se estas evidências como indicativas de atividade do epitélio germinativo foi avaliado se o tipo de ciclo gametogênico contínuo descrito para esta espécie com base em fatores ecológicos e comportamentais (HADDAD \& CARDOSO 1992), também está fundamentado pela morfologia testicular.

Este estudo teve como objetivos específicos: 1) descrever a anatomia das gônadas dos machos de D. minutus através de análises biométricas macroscópicas, 2) descrever a organização histológica geral e 3) caracterizar o tipo de ciclo gametogênico da espécie.

\section{MATERIAL E MÉTODOS}

Cinqüenta exemplares de D. minutus foram capturados mensalmente durante o período de abril de 2004 a março de 2005 (exceto agosto e setembro de 2004, pois os indivíduos não foram observadosem campo, impossibilitando a coleta), por meio de excursões noturnas no Município de São José do Rio Preto São Paulo, Brasil (2045'47,5"S, 4919'38,8"W). Os animais foram mortos e abertos por laparotomia, onde tiveram seus órgãos expostos para as análises. Os testículos foram removidos e medidos em seus maiores eixos com a utilização de paquímetro demetal e, pesados ( $\mathrm{g}$ ) em balança analítica de precisão $(0,001 \mathrm{~g})$. Após este procedimento foram fixados em solução Bouin por 24 horas e desidratados em série crescentes de álcool (Hopwood 1990). Os testículos foram incluídos em resina do tipo M ethacri- lato glicol (Historesin Leica ${ }^{\circledR}$ ) seccionados em 2,0 $\mu \mathrm{m}$ e corados com Azul de Toluidina 1\% (Robinson \& Gray 1990). Foram observados ao microscópio Olympus BX 60 e analisados através do programa Image Pró-plus e Image Tool 3.000. Para a análise da área dos lóculos seminíferos, um total de nove lóculos foi aleatorizado de três animais (três lóculos/animal/mês), totalizando, portanto 90 medidas de área. Quanto ao diâmetro, cinco lóculos foram al eatorizados de cada um dos cinco animais coletados em cada mês ( $n=250)$.

Os dados meteorológicos mensais da temperatura do ar (ㅡ) e pluviosidade ( $\mathrm{mm}$ ) foram concedidos pela Coordenadoria de Assistência Técnica Integral - CATI de São José do Rio Preto, e o fotoperíodo obtido através do site http://www.apolo11.com/ index.php (Fig. 1).
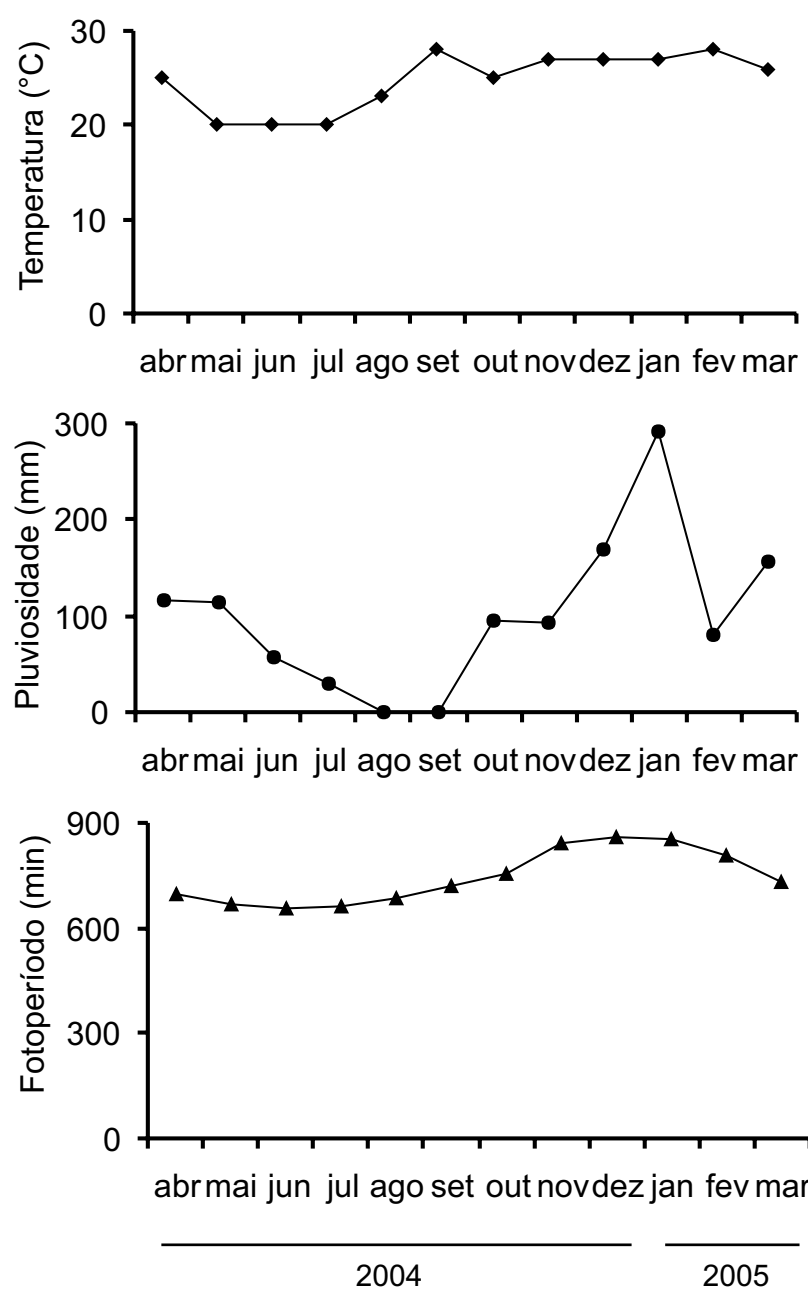

Figura 1. Variação anual na temperatura média (ํㅡ), pluviosidade total (mm) e fotoperíodo (minutos) para a área de São José do Rio Preto, São Paulo, Brasil. Fonte: CATI e site http://www.apolloll. com/index.php.

Revista Brasileira de Zoologia 24 (1): 64-70, março 2007 
Para a análise de dados foi verificada a existência de vaIores discrepantes (outliers), em seguida, a normalidade dos dados foi testada através do teste de Shapiro-Wilk, e quando necessários, foram submetidos à normalização $(x+0,5)^{1 / 2}$ (LEHNER 1996). O comprimento e o peso das gônadas foram analisados entre os meses pelo teste Kruskal-Wallis. Para cada mês, esses parâmetros foram analisados entre a gônada direita e esquerda através do teste Wilcoxon. Para constatar se houve variação dos parâmetros climáticos ao longo do ano foi aplicado o teste Kruskal-Wallis e quando necessário completados pelo teste de Dunn para comparações múltiplas. A área e o diâmetro locular foram analisados pelo teste One-Way ANOVA. A dependência dos parâmetros analisados e as variáveis climáticas foram verificadas por meio do teste de Regressão Linear Múltipla. Foi considerado $p=0,05$ para se atribuir significância estatística, sendo todas as análises baseadas em ZAR (1999).

Os exemplares analisados foram depositados na Coleção Herpetológica do Departamento de Zoologia e Botânica (DZBSJRP), Universidade Estadual Paulista, Instituto de Biociências, Letras e Ciências Exatas de São José do Rio Preto, São Paulo, Brasil.

\section{RESULTADOS}

Machos de $D$. minutus são animais de pequeno tamanho corporal alcançando em média 2,31 0,07 cm de comprimento rostro-cloacal. Os testículos estão localizados na cavidade abdominal, em posição ventral aos rins e apresentam assimetria quanto sua localização. Os rins se encontram em posição imediatamente ventral à parede dorsal desta cavidade e estão intimamente associados aos testículos através do mesentério gonadal ou mesórquio. Os testículos apresentam uma coloração branco-leitosa, possuem forma ovalada, peso médio de $0,002 \pm 0 \mathrm{~g}$ e comprimento médio de 1,90 $\pm 0,13 \mathrm{~mm}$ (Fig. 2) Externamente, os testículos são revestidos por uma cápsula de tecido conjuntivo constituída basicamente por fibras colágenas, que pela sua delgada espessura e transparência, possibilita verificar que os testículos são formados por unidades esféricas, de aspecto granuloso, denominadas lóculos seminíferos (Fig. 3). Estas estruturas abrigam em seu interior as células da linhagem germinativa, com as quais estão associadas às células foliculares ou de Sertoli. Neste epitélio, verificou-se que os machos deD. minutus apresentam diferentes tipos celulares germinativos em seus lóculos seminíferos; as espermatogônias primárias e secundárias normal mente na base do epitélio, os espermatócitos primários e secundários, espermátides iniciais e tardias e os espermatozóides geralmente no lume locular. Ainda quanto à arquitetura histológica testicular, na acomodação dos lóculos seminíferos delimita-se uma área preenchida por tecido intersticial, com vasos sanguíneos e células de Leydig (Fig. 4).

A área e o diâmetro médio do lóculo seminífero são respectivamente de $36012,57 \pm 2128,66 \mu \mathrm{m}^{2}$ e $221 \pm 13 \mathrm{~mm}$. Uma unidade locular seminífera muito similar a estas dimensões obtidas para a espécie esta representada na figura 5.

Revista Brasileira de Zoologia 24 (1): 64-70, março 2007
O comprimento eo peso, entre as gônadas direita $(n=50)$ e esquerda ( $n=50$ ), para cada mês analisado, não apresentou diferença significativa (Wilcoxon, $Z=1,04 ; p=0,29$ ), o que nos permitiu aleatorizar qualquer uma das gônadas para as análises morfométricas da área e diâmetro locular. Adicionalmente foi calculado o índice gonadossomático $(0,07 \pm 0,02 \%)$ o qual estatisticamente não variou ao longo do ciclo $(H=4,86$; $p=0,84)$. O comprimento e o peso das gônadas $(n=100)$ não apresentaram variação entre os meses analisados $(H=11,24$; $\mathrm{p}=0,25$ e $\mathrm{H}=3,71 ; \mathrm{p}=0,92$, respectivamente) (Fig. 6), mas foram positivamente correlacionadas entre si, independentemente do mês (Correlação de Spearman, $r=0,30 ; p=0,03$ ). Estes parâmetros testiculares não manifestaram qualquer relação com as variáveis climáticas analisadas (Regressão linear múltipla, $F=1,32 ; p=0,27$ e $F=0,60 ; p=0,61$, respectivamente) bem como quanto ao comprimento do animal (Correlação de Spearman, $r=-0,01 ; p=0,93$ e $r=-0,02 ; p=0,85$ ).

Quanto a arquitetura histológica, tanto a área (One-Way ANOVA, $F=0,76 ; p=0,65$ ) quanto o diâmetro locular (OneWay ANOVA, $F=0,67 ; p=0,72$ ) não apresentaram variação significativa ao longo do ciclo reprodutivo e não manifestaram qualquer outra associação aos demais parâmetros analisados (Fig. 6).

\section{DISCUSSÃO}

Em vertebrados os testículos têm duas importantes funções: produção de hormônios e a produção de espermatozóides (Hildebrand 1995). Duellman \& Trueb (1994) descrevem os testículos dos anfíbios anuros como estruturas pares, esféricas ou ovais, localizadas numa posição ventral ao nível da metade anterior dos rins, podendo em algumas espécies ser longos e se estenderem até a extremidade posterior dos rins. Segundo GoIn \& Goln (1962) estas estruturas são compactas e de coloração geralmente amarelada.

Os testículos de D. minutus apresentam as mesmas características anatomofisiológicas relatadas para outras espécies de anfíbios anuros: Caudiverbera caudiverbera (Linnaeus, 1758) (Hermosilla et al. 1983), Scinax fuscovarius (Oliveira \& VICENTINI 1998), Eupemphix nattereri (Steindachner, 1863) (OLIVEIRA \& ZIERI 2005). Entretanto, a forma, o pequeno tamanho e o peso dos testículos pode refletir uma característica anatômica associada ao pequeno porte e biótipo do animal. A propriedade de assimetria quanto à localização observada nas gônadas de D. minutus, permite que esta estrutura possa ser deslocada em diferentes alturas, profundidades e orientações de seus eixos Iongitudinal e transversal. Esta mobilidade ocorre em função dos estados fisiológicos das vísceras adjacentes o que pode representar uma característica protetora para a gametogênese (OLIVEIRA \& VICENTINI 1998).

Entretanto no decorrer do ciclo reprodutivo deD. minutus os testículos não manifestaram variação anatômica de tamanho entre os antímeros, ou seja, não há variação intra-individual significativa entre os pares gonadais durante o ciclo. Quan- 


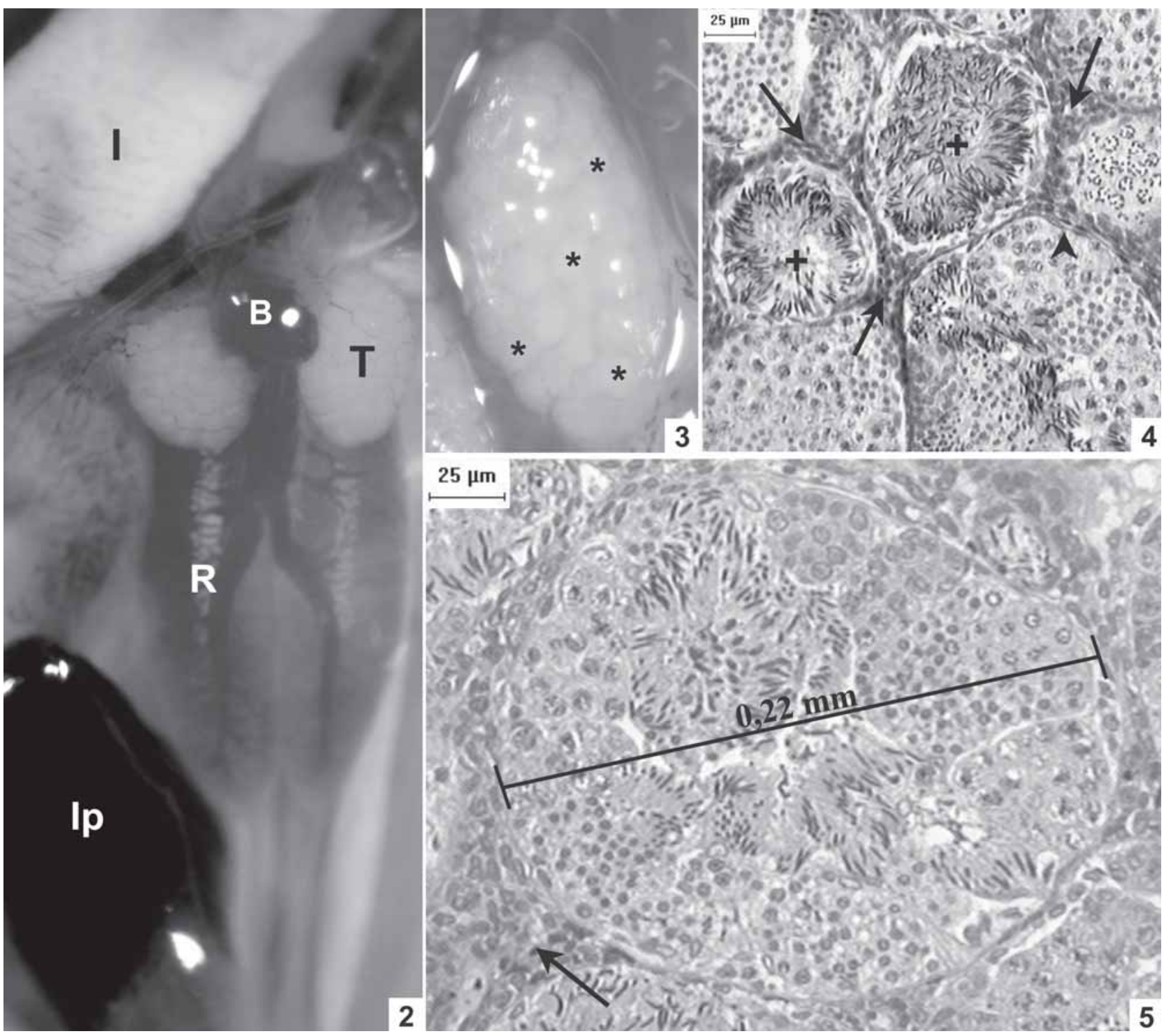

Figuras 2-5. (2) Observação macroscópica dos testículos de Dendropsophus minutus: (B) baço, (I) intestino, (Ip) porção final do intestino com incomum pigmentação conferida por melanócitos viscerais, (R) rim, (T) testículo; (3) detalhe macroscópico dos lóculos seminíferos (*); (4) lóculo seminífero delimitado pela parede locular (cabeça seta), dúctulos eferentes intratesticulares (+) e tecido interlocular (setas); (5) dimensão da estrutura locular que representa a área e o diâmetro médio dos lóculos seminíferos; e o tecido interlocular (setas).

do os indivíduos foram agrupados mensalmente e agrupados ao longo do ciclo, também não houve variação nos parâmetros biométricos, ou seja, o peso e o comprimento não expressam diferenças anatômicas relacionadas a qualquer um dos fatores (fotoperíodo, temperatura e pluviosidade). EMERSON (1997), trabalhando com 90 espécies de anuros, das quais 25 eram representantes da família Hylidae, comparou o peso dos testículos com diferentes sistemas de reprodução, tais como: tamanho da desova, níveis de andrógenos e diferentes graus de comporta- mento mediado por andrógenos e verificou que há diferenças anatômicas dos testículos destes animais. Afirma ainda que esta variação anatômica relaciona-se principalmente à variação na produção de espermatozóides e tamanho da desova em detrimento de comportamentos agonísticos correlacionados com andrógenos. As características anatômicas do testículo de D. minutus são semelhantes às propriedades anatômicas, para outros Hilídeos com biótipo e tamanho corporal semelhante, relatadas por EMERSON (1997). 

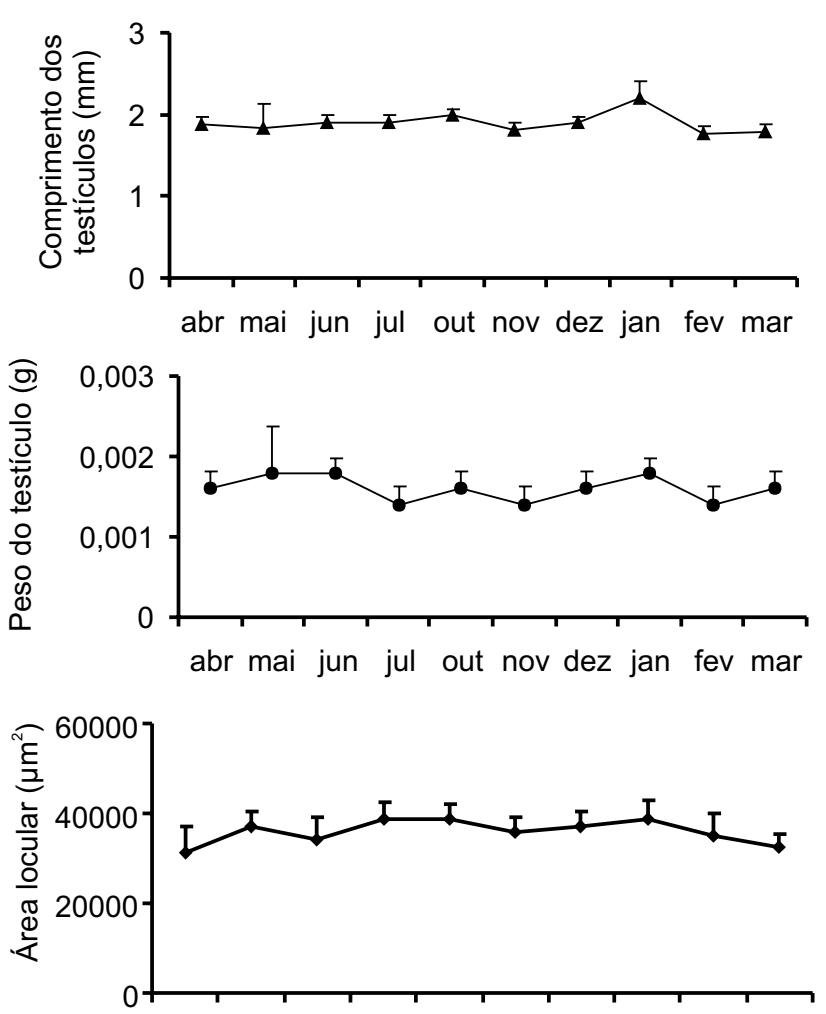

abr mai jun jul out nov dez jan fev mar

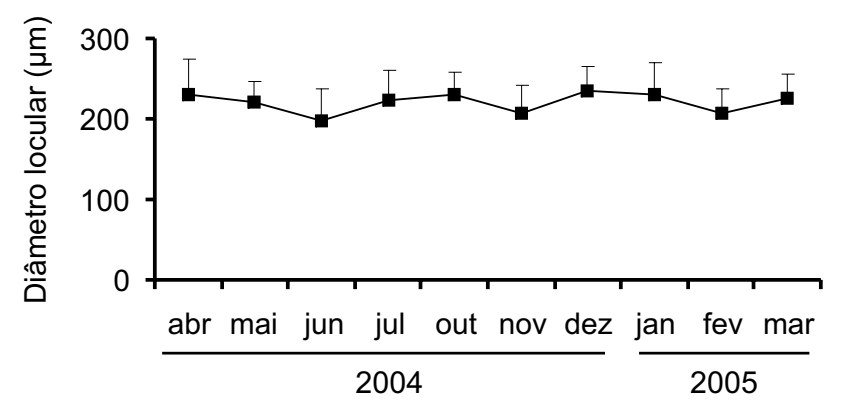

Figura 6. Parâmetros morfométricos: variação do comprimento e peso dos testículos; área e diâmetro dos lóculos seminíferos de Dendropsophus minutus durante o período de abril de 2004 a março de 2005, em São José do Rio Preto, São Paulo. As barras representam o período do estudo.

Segundo Lofts (1974) e HuANG et al. (1997) os ciclos gametogênicos dos anuros, são classificados em tipos descontínuos, potencialmente contínuos e contínuos. Os tipos descontínuos são comumente encontrados em espécies de zonas temperadas e geralmente têm um discreto período de reprodução com pronunciadas mudanças na produção de gametas, no tamanho das gônadas e das estruturas sexuais acessórias, como descrito para Rana dybowskii Günther, 1876 (Ko et al. 1998). O tipo potencialmente contínuo exibe uma parada parcial na atividade espermatogênica durante al gumas estações no ano, mas esper- matogônias primárias permanecem sensíveis à estimulação gonadotrófica, como documentado para Pel ophylax nigromaculatus (Hallowell, 1861) e Glandirana rugosa (Temminck \& Schlegel, 1838) (Ko et al. 1998). Espécies que habitam regiões tropicais, onde as condições climáticas não mostram apreciáveis flutuações, têm um tipo contínuo de ciclo espermatogênico. CALLARD et al. (1978), reporta que no tipo contínuo, ocorrem ondas espermatogenéticas sucessivas, portanto sem interrupção na produção de espermatozóides. Com base nestas considerações e nas análises histológicas dos lóculos seminíferos verificou-se que os machos de D. minutus apresentam tipos celulares diferentes que variam desde espermatogônias primárias aos espermatozóides maduros, em maior ou menor proporção de cada um destes e dos tipos celulares intermediários. Nossos dados corroboram a reprodução contínua ao longo do ano todo previamente descrita para a espécie por HADDAD \& CARDoso (1992) com base em fatores ecológicos e comportamentais, reforçando a ocorrência de espermatogênese contínua, típica para os anfíbios de regiões neotropicais (DuelLman \& TRUeb 1994).

Um aspecto importante a ser salientado é que as variações climáticas não interferem no comprimento e peso dos testículos de D. minutus como também constatado para Pel ophylax perezi (Seoane, 1885) (Delgado et al. 1989). Além disso, em D. minutus não existe variação da área e diâmetro dos lóculos seminíferos nas mesmas condições. Segundo HuANG et al. (1996), anal isando a espécie Bufo bankorensis Barbour, 1908, a variação sazonal na atividade testicular normal mente se reflete no peso gonadal. Tal padrão é geral mente exibido por espécies com um tipo potencialmente contínuo de ciclo espermatogênico e por aquelas com ciclo testicular do tipo descontínuo, tal como Rana temporaria (Linnaeus, 1758), na qual o peso testicular é um índice apropriado da atividade testicular (Lorts et al. 1972). Com base em nossas análises, cabe considerar, portanto, que tanto comprimento e peso testicular quanto diâmetro dos lóculos seminíferos não constituem um bom índice para se atribuir status reprodutivo da espécie em questão, o que pode se aplicar para a maioria dos anuros de regiões neotropicais. Até algumas espécies originárias de áreas temperadas e adaptadas fisiologicamente a regiões neotropicais como Lithobates catesbeianus cujo ciclo gametogênico é contínuo, o peso testicular também não apresentou variação significativa ao longo do ciclo reprodutivo (SASSO-CerRI et al. 2004). Ainda nesse estudo, SASSO-CerRI et al. (2004) sem detectar diferenças macroscópicas, verificaram que há variação no diâmetro do lóbulo seminífero, do tecido intersticial e do volume dos dúctulos excretores, porém, a manutenção do peso testicular em anfíbios que apresentam um ciclo reprodutivo contínuo, pode estar relacionada a mudanças compensatórias no volume e densidade dos componentes testiculares durante o ciclo.

É importante ressaltar que os machos de D. minutus foram identificados vocalizando durante todos os meses. Porém em agosto e setembro, meses em que não choveu, não foram observados em campo, o que nos leva a inferir que esta variá- 
vel climática pode ser um fator controlador da atividade reprodutiva destes animais.

Para D. minutus pequenas oscilações nos parâmetros climáticos locais não implicaria em conseqüentes alterações estruturais tanto em nível macroscópico quanto microscópico das gônadas. No entanto, nesta espécie e em outras com ampla distribuição geográfica, não pode ser refutada a possibilidade desses parâmetros testiculares apresentarem al terações sob condições climáticas geográficas muito adversas.

Informações sobre a biologia reprodutiva (morfologia, modos reprodutivos e aspectos comportamentais) possuem relevante valor biológico e podem ser utilizados para corroborar a filogenia da família, que nos Hylidae foi recentemente revisada (FAivovich et al. 2005).

Em síntese, a análise da anatomia e histologia testicular de D. minutus ao longo da reprodução possibilita concluir que o ciclo gametogênico é contínuo.

\section{AGRADECIMENTOS}

CAPES e Fundação de Amparo à Pesquisa do Estado de São Paulo-FAPESP (processo $n$ o 02/08016-9) pela concessão de bolsa e auxílio financeiro para o desenvolvimento do trabalho. A Giséle Manganelli Fernandes (Departamento de Letras Modernas, IBILCE) pela revisão do abstract e a Thaís Billalba Carvalho pelo auxílio nas análises estatísticas.

\section{REFERÊNCIAS BIBLIOGRÁFICAS}

AichInger, M. 1987. Annual activity patterns of anurans in the seasonal neotropical environment. Oecologia 71 (4): 583592.

BAstos, R.P. \& C.F.B. HAdDAD. 2002. Acoustic and aggressive interactions in Scinax rizibilis (Anura: Hylidae) during the reproductive activity in southeastern Brazil. AmphibiaReptilia 23 (1): 97-104.

Callard, I.P.; G.V. Callard; V. Lance; J.L. Bolaffi \& J.S. Rosset. 1978. Testicular regulation in nonmammalian vertebrates. Biology of Reproduction 18 (1): 16-43.

Delgado, M.J.; P. Gutiérrez \& M. Alonso-Bedate. 1989. Seasonal cycles in testicular activity in the frog, Rana perezi. General and Comparative Endocrinology 73 (1): 1-11.

Duellman, W.E. \& L. Trueb. 1994. Biology of amphibians. New York, McGraw-Hill, 670p.

EMERSON, S.B. 1997. Testis size variation in frogs: testing the alternatives. Behavioral Ecology and Sociobiology 41 (4): 227-235.

Faivovich, J.; C.F.B. Haddad; P.C. Garcia; D.R. Frost; J.A. Cam PBel $\&$ W.C. WheELER. 2005. Systematic review of the frog family Hylidae, with special reference to Hylinae: Phylogenetic analysis and taxonomic revision. Bulletin of the American Museum of Natural History 294: 1-240.

Goln, C.J. \& O.B. GoIn. 1962. Introduction to Herpetology. San Francisco, W.H. Freeman, 341p.
HadDAD, C.F.B. \& A.J. Cardoso. 1992. Elección del macho por la hembra de Hyla minuta (Amphibia: Anura). Acta Zoologica Lilloana 41: 81-91.

HADDAD, C.F.B. \& R.J. SawaYA. 2000. Reproductive modes of Atlantic Forest Hylid frogs: A general overview and the description of a new mode. Biotropica 32 (4b): 862-871.

Hermosilla, B.I.; P.A. Urbina \& P.J.C. Cabrera 1983. Espermatogenesis en la Rana chilena Caudiverbera caudiverbera (Linneaus, 1758) (Anura, Leptodactilidae). Boletin de la Sociedad de Biologia de Concepción 54: 103-115.

Hildebrand, M. 1995. Análise da estrutura dos vertebrados. São Paulo, Atheneu, 700p.

Hoogmoed, M. \& S. GorzulA. 1979. Checklist of the savanna inhabiting frogs of the El Manteco region with notes on their ecology and the description of a new species of tree frogs (Hylidae, Anura), Zoologische Mededelingen 54 (13): 183-216.

Hopwood, D. 1990. Fixation efixatives, p. 21-42. In: J.D. BAnCROFT \& A. STEVEns. Theory and practice of histological techniques. New York, Churchill Livingstone, $3^{\text {rd }}$ ed., 726p.

HuANG, W.S.; J.Y. Lin \& J.Y.L. Yu. 1996. The male reproductive cycle of the toad Bufo bankorensis in Taiwan. Zoological Studies 35 (2): 128-137.

HuANG, W.S.; J.Y. LIN \& J.Y.L. Yu. 1997. Male reproductive cycle of the toad Bufo melanostictus in Taiwan. Canadian Journal of Research: Zoological Sciences 14: 497-503.

Ko, S.K.; H.M. KanG; W.B Im \& H.B. Kwon. 1998. Testicular cycles in three species of Korean frogs: Rana nigromaculata, Rana rugosa and Rana dybowskii. General and Comparative Endocrinology 111: 347-358.

Lehner, P.N. 1996. Handbook of ethological methods. Cambridge, University Press, 672p.

LofTs, B. 1974. Reproduction, p.107-218. In: B. LofTS (Ed). Physiology of the amphibians. New York, Academic Press, 592p.

Lofts, B.; J.J. Wellen \& T.J. BenraAd. 1972. Seasonal changes in endocrine organs of the male common frog, Rana temporaria III. The gonads and cholesterol cycles. General Comparative Endocrinology 18: 344-363.

Oliveira, C. \& C.A. Vicentını. 1998. Descrição anatômica dos testículos e corpos adiposos de Scinax fuscovarius (Anura, Hylidae). Biociências 6 (1): 79-88.

Oliveira, C.; C.A. Vicentini \& S.R. Taboga. 2003. Structural characterization of nuclear phenotypes during Scinax fuscovarius spermatogenesis (Anura, Hylidae). Caryologia 56 (1): 75-83.

Oliveira, C. \& L.R.S. Santos. 2004. Histological characterization of cellular types during Scinax fuscovarius oogenesis (Lutz) (Anura, Hylidae). Revista Brasileira deZoologia 21 (4): 919923.

Oliveira, C. \& R. Zierı. 2005. Pigmentação testicular em Physalaemus nattereri (Steindachner) (Amphibia, Anura) com observações anatômicas sobre o sistema pigmentar extracutâneo.

Revista Brasileira de Zoologia 24 (1): 64-70, março 2007 
Revista Brasileira de Zoologia 22 (2): 454-460.

Paniagua, R.; B. Fraile \& F.J. Sáez. 1990. Effects of photoperiod and temperature on testicular function in amphibians. Histology and Histopathology 5: 365-378.

Rastogi, R.K.; L. Iela; P.K. Saxena \& G. Chieffi. 1976. The control of spermatogenesis of the green frog, Rana esculenta. The Journal of Experimental Zoology 196: 151-166.

Robinson, G. \& T. Gray. 1990. Electron microscopy 2: Tissue preparation, sectioning and staining, p. 525-562. In: J.D. BAnCROFt \& A. SteVens. Theory and practice of histological techniques. New York, Churchill Livingstone, $3^{\text {rd }}$ ed., 726p. Sasso-Cerri, E.; F.P. Faria; E. Freymüller \& S.M. Miraglia. 2004. Testicular morphological changes during the seasonal reproductive cycle in the Bullfrog Rana catesbeiana. Journal of Experimental Zoology 301A: 249-260.

TABogA, S.R. \& M.A.H. Dolder. 1991. Análise histológica da espermatogênese de Hyla ranki (Amphibia, Anura, Hylidae). Revista Brasileira de Ciências Morfológicas 8: 66-71.

ZAR, J. 1999. Biostatistical analyses. New Jersey, Printice Hall, $663 p$.

Recebido em 21.III.2006; aceito em 28.II.2007. 